\title{
Tyrosine kinase inhibitors potentiate the cytotoxicity of MDR-substrate anticancer agents independent of growth factor receptor status in lung cancer cell lines
}

\author{
D. M. Collins • J. Crown • N. O'Donovan • A. Devery • \\ F. O'Sullivan $\cdot$ L. O'Driscoll $\cdot$ M. Clynes $\cdot$ R. O'Connor
}

Received: 9 April 2009 /Accepted: 8 May 2009/Published online: 5 June 2009

(C) Springer Science + Business Media, LLC 2009

\begin{abstract}
Summary To investigate the interactions of Epidermal Growth Factor Receptor (EGFR)-inhibiting tyrosine kinase inhibitors (TKIs) on P-gp-mediated drug resistance, we tested three TKIs, lapatinib, gefitinib and erlotinib in direct ATPase assays and in Non-Small Cell Lung Cancer (NCSLC) cell lines with defined low levels of growth factor receptor expression. The three TKIs potentiated the action of known P-gp substrate cytotoxic drugs at therapeuticallyrelevant concentrations. However, more detailed analysis revealed that the interaction of lapatinib with P-gp was distinct from that of gefitinib and erlotinib, and was characterised by direct inhibition of the stimulated P-gp ATPase activity. Lapatinib proved the most potent P-gp modulator of the TKIs examined. Drug transport studies in the P-gp-over-expressing A549-Taxol cell line showed that lapatinib and erlotinib are capable of increasing docetaxel accumulation at clinically achievable concentrations. Combination studies with P-gp substrate chemotherapeutic
\end{abstract}

Electronic supplementary material The online version of this article (doi:10.1007/s10637-009-9266-0) contains supplementary material, which is available to authorized users.

D. M. Collins $(\bowtie) \cdot$ N. O’Donovan $\cdot$ A. Devery $\cdot$ F. O’Sullivan •

M. Clynes $\cdot$ R. O'Connor

National Institute for Cellular Biotechnology,

Dublin City University,

Glasnevin,

Dublin 9, Ireland

e-mail: denis.collins@dcu.ie

J. Crown

Medical Oncology Department, St. Vincent's University Hospital,

Dublin 4, Ireland

L. O'Driscoll

School of Pharmacy and Pharmaceutical Sciences,

Trinity College Dublin,

Dublin 2, Ireland agents, demonstrated that all three TKIs have significant potential to augment cytotoxic activity against P-gp-positive malignancies, however, interestingly, these agents also potentiated the toxicity of epirubicin in non-P-gp resistant parental cells. Our observations suggest that the combination of lapatinib with a taxane or anthracycline warrants clinical investigation in NSCLC to examine if beneficial or detrimental interactions may result.

Keywords Multi-drug resistance · P-gp · BCRP - MRP-1 . Tyrosine kinase inhibitor · Lapatinib · Erlotinib · Gefitinib . Taxane $\cdot$ Anthracycline

\section{Introduction}

Several ATP-binding cassette $(\mathrm{ABC})$ transporter proteins, including P-gp, BCRP and the multi-drug resistanceassociated protein 1 (MRP-1) have a primary protective physiological function in many areas of the body which are responsible for drug absorption, distribution and elimination, and also in tissue compartments which need special protection from xenobiotic toxicity [1]. However, tumour overexpression of such transporters can likewise be protective for cancer cells, contributing to the emergence of simultaneous tumour resistance to multiple commonly used anti-cancer agents [2]. This phenomenon is typically termed multiple drug resistance (MDR).

P-gp can transport a vast array of structurally unrelated agents, not just cytotoxic drugs, in a unidirectional manner by harnessing a reciprocating ATPase function. The protein possesses multiple sites for drug binding and changes in site conformation and affinity can be induced by drug binding which can affect ATP utilisation, transport activity rate and P-gp interactions with other drug substrates [3]. 
Lung cancer is a molecularly heterogeneous malignancy, however, overexpression of drug transport proteins, especially P-gp, is a common observation of clinical and laboratory specimens and is a likely contributor to the high incidence of drug resistance in this disease [4].

Despite recognition of the important role of P-gp in lung cancer and other commonly drug resistant malignancies and the many attempts to develop pharmacological inhibitor strategies, no randomised Phase III drug trials of P-gp circumvention combined with cytotoxic administration have demonstrated the anticipated increase in anti-tumour action. The current third generation of P-gp inhibitors, such as elacridar and tariquidar, are potent but their clinical application has been confounded by unfavourable pharmacokinetic interactions with P-gp substrate drugs, in particular serum level elevations of the co-administered cytotoxic caused by an inhibition of P-gp-mediated drug elimination from normal body tissues. [5].

Cancer chemotherapy treatment is being revolutionised by the advent of less toxic, molecularly-targeted cancer drugs. These agents interact with over-expressed or mutated oncogenic target pathways which have been described in many cancers and can display impressive selective anti-tumour activity [6]. One protein family which has been a major focus for molecularly-targeted drug development is the erbB family of type I receptor tyrosine kinases which consist of EGFR (epidermal growth factor receptor/erbB1/Her-1), HER-2 (erbB2/neu), erbB3 (Her-3) and erbB4 (Her-4). These receptors play an integral role in normal cell growth and differentiation [7]. Several molecularly targeted therapies which target the erbB family have been approved for cancer treatment while others are currently in development [8]. Overexpression of normal or mutated EGFR and HER-2 is found in many malignancies including non-small cell lung cancer (NSCLC), breast and colon cancers, and is often associated with poorer prognosis or reduced response to chemotherapy [9]. Recent studies have demonstrated improved treatment efficacy using combinations of cytotoxic drugs with molecularly targeted agents and it seems likely that the range of such combinations will continue to increase in the future [10].

Gefitinib and erlotinib are EGFR-targeted small molecule tyrosine kinase inhibitors (TKIs) while lapatinib is the first dual inhibitor of EGFR and HER-2 tyrosine kinases to reach marketing approval [8]. Gefitinib has demonstrated some activity in the treatment of a subpopulation of patients with NSCLC that have specific EGFR mutations, while erlotinib has prolonged survival in patients with NSCLC after the failure of first or second line chemotherapy [11]. Lapatinib has shown clinical benefit in metastatic trastuzumab-refractory HER-2positive breast cancer when administered in combination with capecitabine, and has recently received FDA approval for use in this setting [12].

The ability of gefitinib and erlotinib to interact with another $\mathrm{ABC}$ transporter, the Breast Cancer Resistance Protein, BCRP, (ABCG2) in vitro has been reported previously [13-15]. Kitazaki et al. [16], also demonstrated that gefitinib could synergise the in vitro toxicity of $\mathrm{P}$-gp substrate-cytotoxics in P-gp overexpressing cells through inhibition of the pump. Shi et al., examined the interactions of erlotinib with P-gp in drug resistant cancer cells and showed that erlotinib could also synergise P-gp substrate cytotoxic actions through P-gp inhibition [17]. Lapatinib has recently been reported to interact with P-gp and BCRP [18].

We have undertaken a comparative study of all three EGFR-inhibiting TKIs in human lung cancer cell models, with different levels of P-gp and low or varying growth factor receptor expression, in order to assess their potential for MDR reversal in vitro, the mechanistic basis of such interactions and the potential pharmacological implications for concurrent usage of these agents with standard chemotherapeutic agents.

\section{Materials and methods}

Cell lines, cell culture and reagents

All cell lines were cultured at $37^{\circ} \mathrm{C}$ without antibiotics and with routine monitoring for mycoplasma contamination. A549, an adherent lung adenocarcinoma cell line was cultured in DMEM/Ham F10 supplemented with 5\% FCS. A549-Taxol, a drug resistant variant of A549 selected by ten pulses $\left(4 \mathrm{hrs} /\right.$ week) with paclitaxel $\left(\operatorname{Taxol}^{\circledR}\right)(50 \mathrm{ng} / \mathrm{ml})$, was cultured in identical conditions.

DLKP is an adherent, poorly differentiated human squamous cell lung carcinoma cell line grown in DMEM/ Ham F10 supplemented with 5\% FCS [19]. DLKP-A is a drug resistant variant of DLKP selected by increasing exposure to adriamycin [20].

Erlotinib and gefitinib were obtained from Sequoia Research Chemicals Ltd. (Pangbourne, U. K.). Lapatinib ditosylate and elacridar were obtained from GlaxoSmithKline (Middlesex, U. K.). All were dissolved in DMSO. Cyclosporin $\mathrm{A}$ and all other reagents, unless specified otherwise, were purchased from Sigma-Aldrich (Dublin, Ireland). ${ }^{14} \mathrm{C}$ radiolabelled docetaxel (specific activity, $60 \mathrm{mCi} / \mathrm{mmol}$, dissolved in ethanol) was purchased from American Radiolabelled Chemicals, Inc. (St. Louis, MO. U.S.A.). The clinical formulations of docetaxel, Taxotere ${ }^{\circledR}$ (Sanofi-Aventis, Bridgewater, NJ, U.S.A.), paclitaxel, Taxol $^{\circledR}$ (Bristol Myers Squibb, Uxbridge, Middlesex, U.K.), Ellence $^{\circledR}$ (epirubicin hydrochloride) (Pfizer, Cambridge, MO, 
USA), and Cisplatin (Mayne Pharma Plc., Warwickshire, U.K.) were utilised in proliferation assays.

\section{Western blot}

For detection of P-gp, BCRP and MRP-1, protein samples were prepared with approx. $30 \mu \mathrm{g}$ protein in $10 \mu \mathrm{l}$ sample buffer (RIPA buffer, protease inhibitor, PMSF and sodium orthovanadate) plus $10 \mu \mathrm{l}$ of $2 \times$ loading buffer. Samples were not denatured before loading. Samples were examined by SDS-PAGE gel electrophoresis using a $7.5 \%$ gel. The primary antibodies used were P-gp (MDR1, ABCB1) mouse monoclonal IgG2 (Santa Cruz Biotechnology, CA., U.S.A), BCRP (ABCG2) mouse monoclonal IgG2 antibody (Alexis Biochemicals, UK) and.MRP-1 (ABCC1) mouse monoclonal IgG1 (Santa Cruz Biotechnology, CA., U.S.A) $\alpha$-tubulin and $\beta$ - actin primary antibody and mouse secondary antibody was obtained from Sigma-Aldrich (Dublin, Ireland). Luminol detection reagent (Santa Cruz Biotechnology Inc.,CA., U.S.A) was used to visualise the protein bands.

\section{ELISA}

Quantification of EGFR was carried out using a DuoSet ELISA Development kit from RandD Systems Europe, Ltd. (Abingdon, U.K.). HER-2 quantification was carried out using HER-2 ELISA kit from Calbiochem (Merck Chemicals Ltd., Nottingham, U.K.). Assays were read on a Labsystems Multiskan Ex at $450 \mathrm{~nm}$ with wavelength correction at $570 \mathrm{~nm}$.

\section{ATPase studies}

P-gp and MRP-1 ATPase studies were carried out using purified membrane vesicles from Sf9 (Spodoptera frugiperda) (Solvo Biotechnology, Budapest, Hungary). BCRP ATPase activity was assessed in membrane vesicles purified from a selected, BCRP over-expressing mammalian cell line (Solvo Biotechnology, Budapest, Hungary). The protocols provided by Solvo Biotechnology are a modification of the method of Sarkadi et al., 1992 [21]. Two variants of this assay were employed in our studies.

The activation assay measured the increase in vanadatesensitive ATPase activity in the presence of a range of test compound concentrations through spectrophotometric quantitation of the amount of inorganic phosphate generated as a product of ATPase-mediated conversion of ATP to ADP. This assay gave a measure of the ability of an agent to stimulate transporter activity (from basal levels), a characteristic common to substrates of such ATPase transporters.

In the inhibition assay, ATPase activity is maximally stimulated using a saturating concentration of an activator of the transporter being studied. P-gp ATPase activity was stimulated with $40 \mu \mathrm{M}$ verapamil and MRP-1 ATPase activity stimulated by $10 \mathrm{mM}$ n-ethyl maleimideglutathione (NEM-GS) mix, and the decrease in this maximum vanadate-sensitive P-gp ATPase activity was measured in the presence of a range of test compound concentrations. Agents which inhibit P-gp or MRP-1 function will typically reduce the ATPase activity of the pump, leading to a decrease in the activator-stimulated production of inorganic phosphate. All MRP-1 ATPase studies were carried out in the presence of $2 \mathrm{mM}$ glutathione.

According to the supplied protocol, the basal level of BCRP ATPase activity in the membrane vesicles is much higher than P-gp or MRP-1 ATPase allowing measurement of any direct inhibition of BCRP ATPase activity by a test compound without the use of an activator. Maximal stimulation of BCRP ATPase was achieved with $10 \mu \mathrm{M}$ sulfasalazine and maximal inhibition of BCRP ATPase was achieved with $10 \mu \mathrm{M}$ Hoechst 33342. Assays were read on a Molecular Devices SPECTRAmax PLUS 384 spectrometer at $650 \mathrm{~nm}$.

${ }^{14} \mathrm{C}$ radiolabelled docetaxel transport assays

\section{${ }^{14} \mathrm{C}$ radiolabelled docetaxel accumulation assay}

Wells of a tissue culture-treated 24-well plate (Costar Cat\# 3524, Corning Inc., Corning, NY 14831, U.S.A.) were seeded with $1 \mathrm{ml}$ of cell suspension containing $1 \times 10^{5}$ cells per ml. Following a $24 \mathrm{~h}$ incubation at $37^{\circ} \mathrm{C}$, medium was removed from the cells. Three wells received $250 \mu \mathrm{l}$ medium, 15 wells received the test compound at various concentrations and three wells received the vehicle control. $500 \mu 1$ medium was placed in the remaining three wells required for background counts. $250 \mu \mathrm{l} 2 \times 100 \mathrm{nM}$ (or $500 \mathrm{nM}){ }^{14} \mathrm{C}$ docetaxel was then added to the 21 test wells. Drug-containing medium was removed after $90 \mathrm{~min}$ and each well was washed twice with cold PBS followed by the addition of $250 \mu 11 \mathrm{M} \mathrm{NaOH}$. Samples were then transferred to scintillation vials containing $3 \mathrm{ml}$ scintillation fluid to be analysed with a Beckman LS6500 multi-purpose scintillation counter (Beckman-Coulter, Inc., CA., U.S.A). Medium was removed from the three remaining wells on each plate, the cells were trypsinised, and a cell count performed. All medium used in the assay was pre-warmed to $37^{\circ} \mathrm{C}$.

\section{${ }^{14} \mathrm{C}$ radiolabelled docetaxel efflux assay}

Four 24-well plates (A,B,C, and D) were seeded with $1 \mathrm{ml}$ of cell suspension containing $1 \times 10^{5}$ cells per $\mathrm{ml}$ and incubated at $37^{\circ} \mathrm{C}$ for $24 \mathrm{~h}$. Medium was removed and replaced with $500 \mu \mathrm{l}$ medium containing ${ }^{14} \mathrm{C}$ radiolabelled docetaxel at a concentration of $500 \mathrm{nM}$. The plates were incubated at $37^{\circ} \mathrm{C}$ for $90 \mathrm{~min}$. The drug containing medium 
was then removed. The cells in Plate A, the 90 min control, were washed twice in cold PBS and $250 \mu 1$ of $1 \mathrm{M} \mathrm{NaOH}$ was added. Pre-warmed medium, three concentrations (1, 5 and $10 \mu \mathrm{M})$ of test drug and a DMSO control were then added to plates B, C and D in triplicate. Drug was removed from one of the plates at 20,40 and $60 \mathrm{~min}$ respectively, following the procedure outlined for plate A. A cell count was also carried out for each plate. Samples were then transferred to scintillation vials containing $3 \mathrm{ml}$ scintillation fluid to be analysed. Results are expressed as the counts per minute (CPM) values per 10,000 of cells.

Laser scanning confocal microscopy

Cells were seeded at $1 \times 10^{5}$ cells/well on glass coverslip chamber slides (NUNC, Roskilde, Denmark) $24 \mathrm{~h}$ prior to the experiment. Cells were exposed to $2 \mu \mathrm{M}$ epirubicin alone or in combination with tyrosine kinase inhibitor for $120 \mathrm{~min}$. Drug was removed and replaced with medium. A spectral scan was performed to obtain the optimum experimental excitation and emission wavelengths. Epirubicin fluorescence was examined in situ by exciting at $514 \mathrm{~nm}$ and visualising the emitted light collected between 531-667 nm (Em (max) $600 \mathrm{~nm}$ ) by confocal laser scanning microscopy (Leica TCS AOBS, Leica Microsystems GmbH, Wetzlar, Germany).

\section{Cell proliferation assays}

The anti-proliferative effects of drugs alone $\left(\mathrm{IC}_{50}\right)$ and in combination were measured using a 7 -day acid phosphatasebased proliferation assay as described in reference [22]. Cells were seeded in a 96 well-plate at a concentration of $1 \times 10^{3}$ cells per well. The cells were left to attach overnight in a $5 \% \mathrm{CO}_{2}$ incubator at $37^{\circ} \mathrm{C}$. For $\mathrm{IC}_{50}$ determinations, $100 \mu \mathrm{l}$ of a range of $2 \times$ drug concentrations were added after $24 \mathrm{~h}$. For combination studies, the two compounds to be examined were added in $50 \mu 1$ of medium at a $4 \times$ concentration. Plates were incubated in the aforementioned conditions until termination of the assay on day 7. All assays were performed in triplicate plates. The drug-containing medium was removed from the 96-well plates. Each well was washed using PBS. The PBS was then removed and $100 \mu 1$ of sodium acetate buffer containing acid phosphatase substrate added at a concentration of $0.263 \mathrm{~g}$ acid phosphatase substrate $(\mathrm{PnP})$ per $100 \mathrm{ml}$ buffer. The plates were transferred to a $37^{\circ} \mathrm{C}$ incubator for $75 \mathrm{~min}$. The reaction was stopped by addition of $50 \mu 11 \mathrm{M} \mathrm{NaOH}$. Plates were read at $405 \mathrm{~nm}$ with $620 \mathrm{~nm}$ as the reference wavelength.

Statistical analysis of data

Comparative statistical analyses were performed on the quantitative data generated in the study. Statistical analysis was performed using Microsoft Excel ${ }^{\circledR}$. Where appropriate, differences between experimental group mean values were evaluated by two tailed student's t-tests and considered significant if $\mathrm{P}<0.05$. All error values represent the standard deviation (SD) of the mean.

\section{Results}

\section{P-gp, BCRP, MRP-1 expression}

The levels of P-gp, BCRP and MRP-1 in the DLKP, DLKPA, A549 and A549-Taxol cell lines were determined by Western blotting (Fig. 1). The parent DLKP and A549 cell lines did not express detectable levels of P-gp but the adriamycin-selected, DLKP-A, and taxol-selected, A549Taxol, cell lines over-expressed P-gp. A549 and A549Taxol expressed MRP-1, DLKP and DLKP-A did not. All four cell lines examined did not exhibit BCRP expression.

EGFR and HER-2 status of the cell lines

An ELISA was required to detect the low levels of EGFR and HER-2 in the lung cancer cell lines (Supplementary Fig. 1). All four cell lines expressed low HER-2 levels (DLKP $1.8 \pm 1.4 \mathrm{pg} / \mu 1$, DLKP-A $1.1 \pm 0.7 \mathrm{pg} / \mu 1$, A549 $0.8 \pm$ $0.7 \mathrm{pg} / \mu \mathrm{l}, \mathrm{A} 549$-Taxol $0.7 \pm 0.4 \mathrm{pg} / \mu \mathrm{l})$. DLKP was EGFRnegative while DLKP-A $(0.8 \pm 0.2 \mathrm{pg} / \mu \mathrm{l})$ showed low EGFR expression. A549 $(33.4 \pm 2.3 \mathrm{pg} / \mu \mathrm{l})$ and A549Taxol $(24.1 \pm 2.4 \mathrm{pg} / \mu \mathrm{l})$ both expressed significantly higher EGFR levels than DLKP-A.

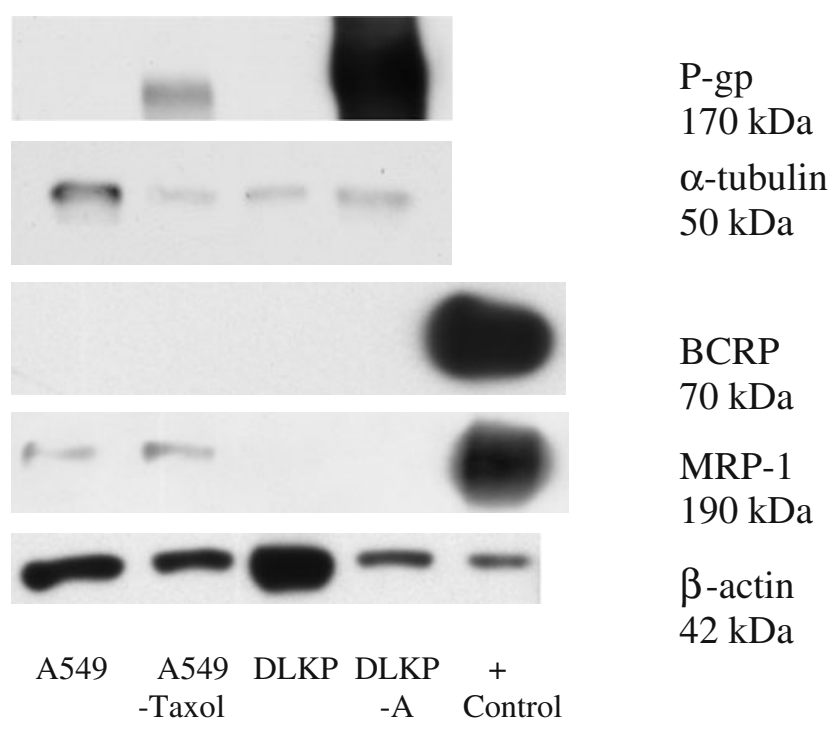

Fig. 1 Western blot for P-gp (170 kDa), BCRP (70 kDa) and MRP-1 (190 kDa), $\alpha$-tubulin (50 kDa) expression was used as a control for P-gp expression while $\beta$-actin was used for BCRP and MRP-1 expression. Positive controls were DLKP-SQ-Mitox (BCRP) and 2008-MRP1 (MRP-1) 
Drug toxicity assays with the TKIs (EGFR and/or HER-2 inhibitors) used in the study also indicated that none of the cell lines chosen displayed sensitivity to growth factor receptor inhibition $\left(\mathrm{IC}_{50}\right.$ values were all $>2 \mu \mathrm{M}$, Table 1).

\section{Effects of TKIs on transporter ATPase activity}

We compared all three TKIs and the classic P-gp inhibitor, cyclosporin A, in P-gp ATPase inhibition (Fig. 2a) and activation (Fig. 2b) assays to determine and compare the ability of these agents to interact with P-gp and to better characterise their mechanism of P-gp modulation. In the inhibition assays (which characterise an agents ability to inhibit stimulated P-gp activity), lapatinib displayed direct inhibition of verapamil-activated P-gp ATPase activity. However, as anticipated, the potent P-gp inhibitor, cyclosporin A, demonstrated the greatest inhibitory effect at low concentrations. Erlotinib and gefitinib did not reduce verapamil-induced P-gp ATPase activity and, in fact, gefitinib stimulated P-gp ATPase activity above the control stimulated levels.

In the activation assay, (used as a measure of how good a particular agent is as a substrate), all three TKIs displayed activation of P-gp ATPase activity at low concentrations. At higher concentrations $(10-40 \mu \mathrm{M})$, gefitinib and erlotinib were strong activators of P-gp ATPase activity. In the case of lapatinib, the small amount of activation evident at low concentrations plateaued and declined with drug concentrations above $5 \mu \mathrm{M}$. Cyclosporin A was the weakest activator of P-gp, consistent with its being a good inhibitor but a poor substrate of P-gp.

Sulindac, an MRP-1 modulator [23], was the only compound that stimulated MRP-1 ATPase activity, Fig. 2c. All three TKIs examined exhibited minor activity by continuously increasing MRP-1 ATPase activity with increasing concentration but their effects did not differ significantly from each other, Fig. 2c. An MRP-1 ATPase inhibition assay revealed that none of the TKIs significantly altered NEM-GS-stimulated MRP-1 ATPase activity (Supplementary data Fig. 2). Results indicated that all three TKIs were poor activators and inhibitors of MRP-1 ATPase activity.

The high basal activity levels of BCRP ATPase allowed both activation and inhibition to be measured directly, Fig. 2d. Results showed gefitinib to be the most potent stimulator of BCRP ATPase activity, followed by erlotinib and then lapatinib. All three TKIs stimulated ATPase activity at low concentrations $(0.25-5 \mu \mathrm{M}$ for gefitinib and erlotinib, $0.25-2.5 \mu \mathrm{M}$ for lapatinib). Above these concentrations the stimulatory effect began to decrease and in the case of lapatinib, the agent inhibited basal ATPase activity above $5 \mu \mathrm{M}$. The BCRP-substrate mitoxantrone decreased BCRP ATPase below baseline levels at all concentrations. Elacridar, a potent third generation P-gp and BCRP inhibitor, decreased BCRP ATPase activity below the maximal inhibited value achieved by $0.1 \mathrm{mM}$ Hoechst 33342 at all concentrations examined, proving it to be a superior inhibitor of BCRP ATPase activity. Results suggested that gefitinib and erlotinib were better activators of BCRP ATPase activity than lapatinib at low concentrations.

TKI-related alterations in docetaxel accumulation and efflux in the P-gp+ DLKP-A cell line

A radiolabelled docetaxel accumulation assay was employed to examine the implications of P-gp modulation on the efflux of this cytotoxic P-gp substrate. The activities of gefitinib, erlotinib and lapatinib were compared with cyclosporin A and elacridar, in the P-gp over-expressingDLKP-A cell line.

Gefitinib and erlotinib increased docetaxel accumulation in a concentration-dependent manner comparable to cyclosporin A (Fig. 3) while, surprisingly, lapatinib proved even

Table $1 \quad \mathrm{IC}_{50}$ values $(\mu \mathrm{M})$ determined from 7-day proliferation assays

\begin{tabular}{lcccc}
\hline Drug & DLKP & DLKP-A & A549 & A549-Taxol \\
\hline Docetaxel & $0.00038 \pm 0.000015$ & $0.087 \pm 0.0067 \mathbf{( 2 2 8 )}$ & $0.00025 \pm 0.000021$ & $0.0014 \pm 0.00054 \mathbf{( 5 . 6 )}$ \\
Paclitaxel & $0.002 \pm 0.0001$ & $0.31 \pm 0.013 \mathbf{( 1 5 3 )}$ & $0.0027 \pm 0.00057$ & $0.0098 \pm 0.00066(\mathbf{3 . 6})$ \\
Epirubicin & $0.017 \pm 0.0008$ & $2.1 \pm 0.24 \mathbf{( 1 2 3 )}$ & $0.038 \pm 0.0045$ & $0.03 \pm 0.0047(\mathbf{0 . 8})^{\dagger}$ \\
Cisplatin & $1.1 \pm 0.24$ & $2.0 \pm 0.17 \mathbf{( 1 . 9 )}$ & $2.4 \pm 0.44$ & $\left.2.6 \pm 0.23 \mathbf{( 1 . 1}^{\dagger}\right)$ \\
Lapatinib & $2.7 \pm 0.1$ & $\left.2.4 \pm 0.2 \mathbf{( 0 . 9}^{\dagger}\right)$ & $2.8 \pm 0.1$ & $3.7 \pm 0.1(\mathbf{1 . 3})$ \\
Gefitinib & $6.1 \pm 0.02$ & $8.5 \pm 0.36 \mathbf{( 1 . 4 )}$ & $7.3 \pm 0.33$ & $7.4 \pm 0.23\left(\mathbf{1}^{\dagger}\right)$ \\
Erlotinib & $8.8 \pm 0.5$ & $11.1 \pm 0.5 \mathbf{( 1 . 3 )}$ & $3.1 \pm 0.2$ & $9.3 \pm 1.8(\mathbf{3})$ \\
\hline
\end{tabular}

Values represent the average of three independent determinations. Fold increase in resistance compared to the parent cell line is given in brackets. All values were significant except those highlighted where $\mathrm{P}$ value found to be greater than 0.05

(† values, not significant, $P>0.05$ ) 

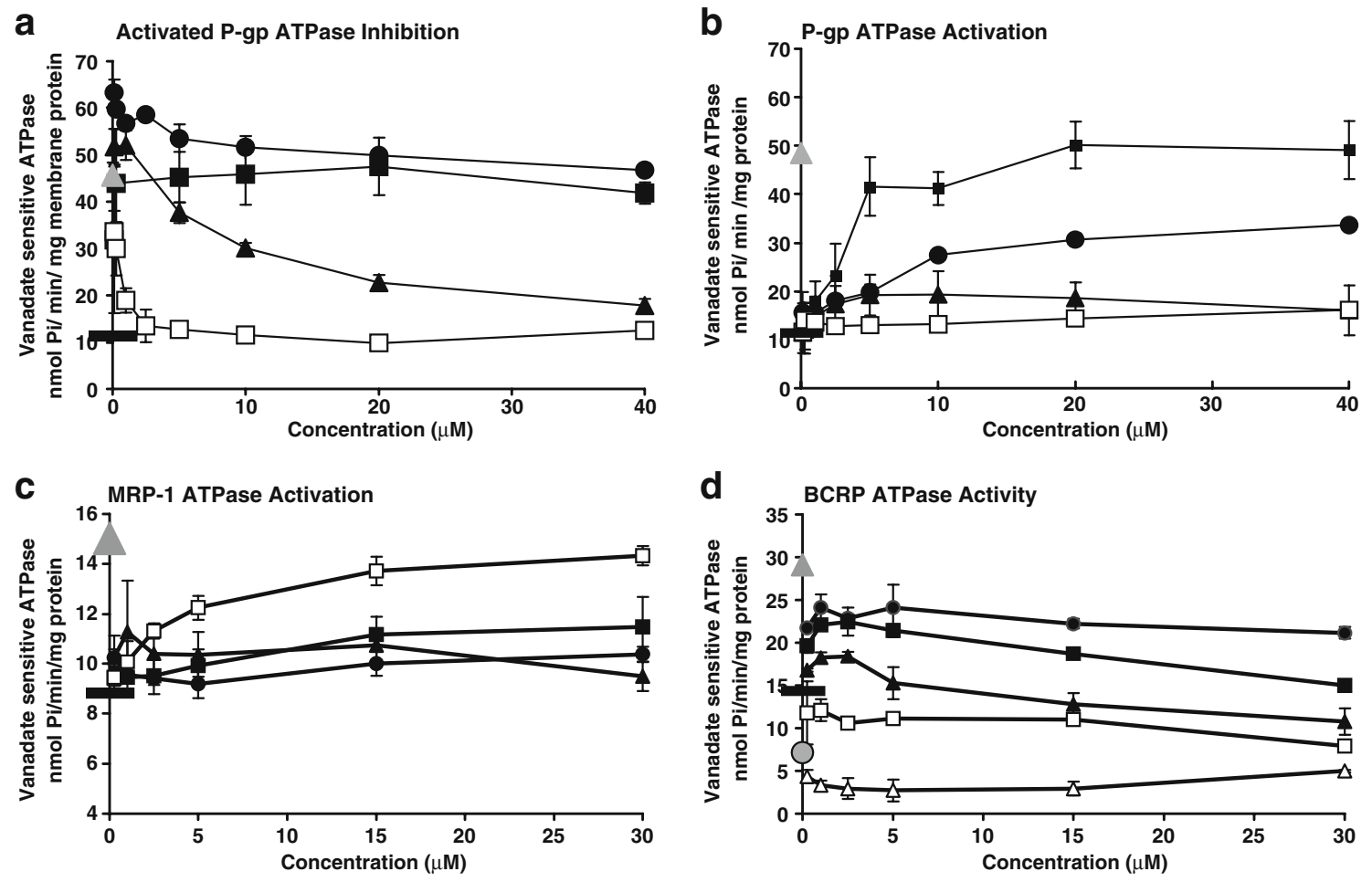

Fig. 2 The effects of lapatinib $(\boldsymbol{\Delta})$, gefitinib $(\bullet)$ and erlotinib $(\boldsymbol{\bullet})$ on vanadate-sensitive transporter ATPase activity. Baseline (-), maximal stimulated $(\triangle)$ and maximal inhibited $(\bigcirc)$ ATPase levels are shown. Agents used to generate maximal stimulation and inhibition can be found in Materials and Methods. For the P-gp ATPase inhibition study (a), $40 \mu \mathrm{M}$ verapamil was included in all tests. P-gp ATPase activation (b) measured ATPase activity in the absence of a stimulating agent. Cyclosporin A ( $\square$ ) was the reference compound for (a) and (b). For

more effective at increasing docetaxel levels than cyclosporin A. The non-competitive P-gp inhibitor, elacridar, produced the most potent increase in docetaxel accumulation. Lapatinib, erlotinib, gefitinib, cyclosporin A and elacridar, at equal concentrations $(1 \mu \mathrm{M})$, each increased docetaxel accumulation 4.2, 1.6, 1.6, 2.1, and 6.3 fold, respectively.

MRP-1 ATPase activation (c), sulindac ( $\square$ ) acted as reference compound. For BCRP ATPase activity (d), the reference compound was mitoxantrone $(\square)$ while elacridar $(\Delta)$ was included as a reference inhibitor. (a), (b) and (c) utilised $S f 9$ insect cell membrane preparations, (d) utilised cell membrane preparations of mammalian origin. All compounds were dissolved in DMSO except cyclosporin A which was dissolved in ethanol. Each concentration was determined in duplicate

Gefitinib, lapatinib or erlotinib $(10 \mu \mathrm{M})$ did not increase docetaxel accumulation over $90 \mathrm{~min}$ in the P-gp-negative DLKP cell line (data not shown).

To prove that these effects on taxane accumulation were due to inhibition of P-gp-mediated efflux, radiolabel experiments were also conducted to specifically measure the impact of TKI co-administration on docetaxel efflux

Fig. 3 Accumulation of $100 \mathrm{nM}{ }^{14} \mathrm{C}$ radio-labelled docetaxel in DLKP-A over $90 \mathrm{~min}$. Values represent the average of three determinations. All inhibitors were dissolved in DMSO, except cyclosporin A (ethanol). († not significant relative to $0.1 \mu \mathrm{M}$ docetaxel control, $P>0.05$, all other points significant relative to $0.1 \mu M$ docetaxel control, $P<0.05$ )

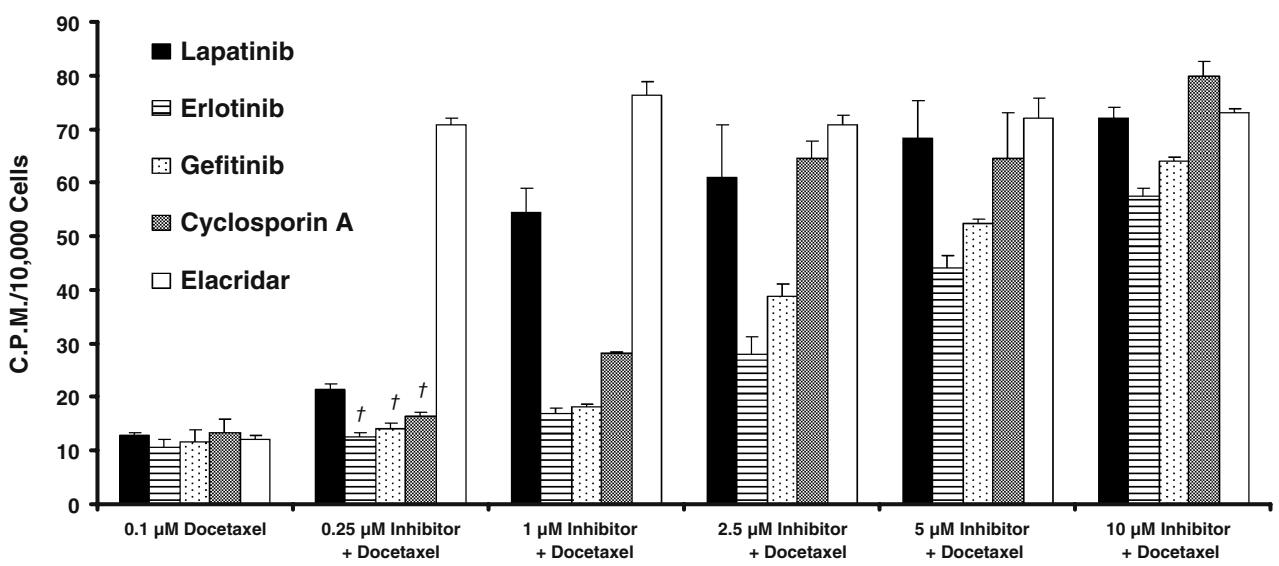


in the P-gp-overexpressing DLKP-A cells. Again, lapatinib and elacridar were the most potent inhibitors of docetaxel efflux, producing significant decreases in docetaxel efflux at the lowest concentration examined (Fig. 4).

Increased epirubicin accumulation in the DLKP-A cell line

Accumulation of the naturally fluorescent P-gp substrate chemotherapeutic, epirubicin, was examined in DLKP-A. Laser scanning confocal imaging was utilised to visualise the cellular distribution and increased epirubicin accumulation in DLKP-A (Fig. 5 a-f). After 120 min exposure to $2 \mu \mathrm{M}$ epirubicin, only minute cytoplasmic levels of the drug were visible (a), a similar result to the DMSO control (f). Due to TKI-mediated P-gp inhibition, there was an increase in fluorescence visible in the presence of $5 \mu \mathrm{M}$ lapatinib (b), erlotinib (c), gefitinib (d) and the P-gp inhibitor elacridar (e). In particular, the level of nuclear fluorescence increased.

Examination of the toxicological implications of TKI-mediated P-gp inhibition in the presence of substrate cytotoxics

$\mathrm{IC}_{50}$ values for the chemotherapy agents docetaxel, paclitaxel, epirubicin and cisplatin and the TKIs, lapatinib, gefitinib and erlotinib are provided in Table 1.
P-gp over-expression in DLKP-A resulted in a 228, 153 and 123-fold resistance to docetaxel, paclitaxel and epirubicin, respectively, compared to the parent cell line. Resistance to the non-P-gp substrate, cisplatin, was 1.9-fold in comparison. The lower level of P-gp expression in A549Taxol corresponded with lower fold increases in resistance to docetaxel (5.6) and paclitaxel (3.6). Interestingly, there was no increase in epirubicin $(0.8)$ resistance. On the whole, at the high concentrations necessary to produce a toxic effect in these growth factor receptor inhibitor insensitive cells, the Pgp expression in DLKP-A and A549-Taxol cells lead to very minor decreases in sensitivity to the TKIs used.

Peak plasma drug concentrations $\left(\mathrm{C}_{\max }\right.$ values $)$ for therapeutic doses of gefitinib $(225 \mathrm{mg})$, erlotinib $(150 \mathrm{mg})$ and lapatinib $(1200 \mathrm{mg})$ have been determined at approximately $0.7 \mu \mathrm{M}, 4 \mu \mathrm{M}$, and $2 \mu \mathrm{M}$, respectively [24-26]. TKI concentrations close to pharmacological levels and providing low cytotoxicity were selected for combination studies. A supra-additive decrease in cell survival resulted from co-treatment with the TKIs and the P-gp substrates docetaxel and epirubicin in the high P-gp-overexpressing DLKP-A cell line (Table 2). Similar results were seen in the P-gp -positive A549-Taxol cell line (Table 3). No potentiation of toxicity was observed when the TKIs were combined with the non-P-gp substrate cisplatin (Supplementary data Table 1). Of the three TKIs examined, lapatinib exhibited the most effective potentiation of docetaxel and epirubicin toxicity at the lowest concentrations.

\section{DLKP-A}

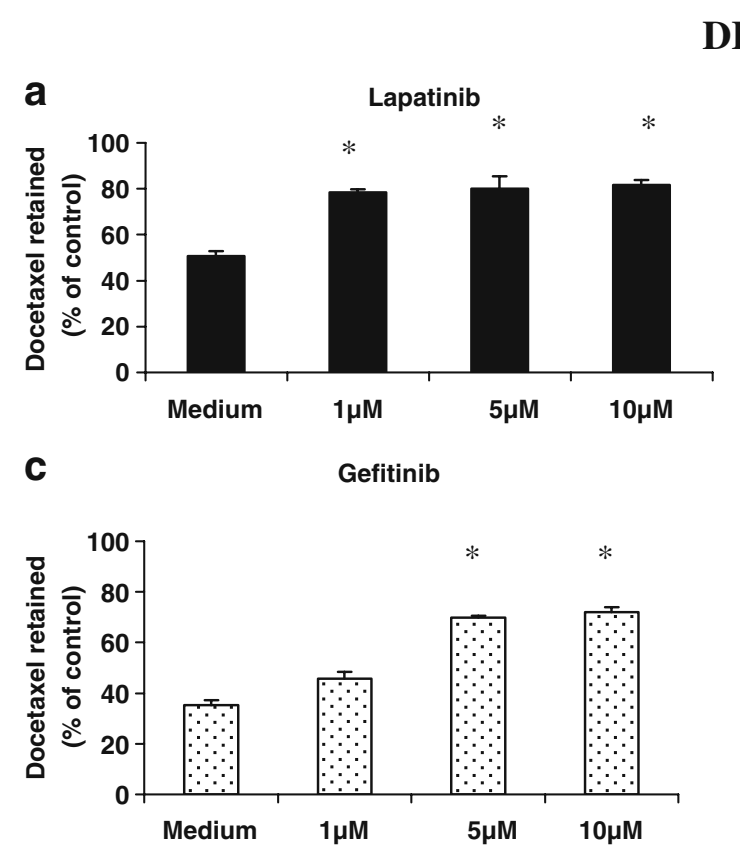

Fig. 4 Efflux of ${ }^{14} \mathrm{C}$ radio-labelled docetaxel from DLKP-A. Cells were exposed to $0.5 \mu \mathrm{M}{ }^{14} \mathrm{C}$ radiolabelled docetaxel for $90 \mathrm{~min}$ (Control), the drug removed and replaced with medium alone or a concentration of

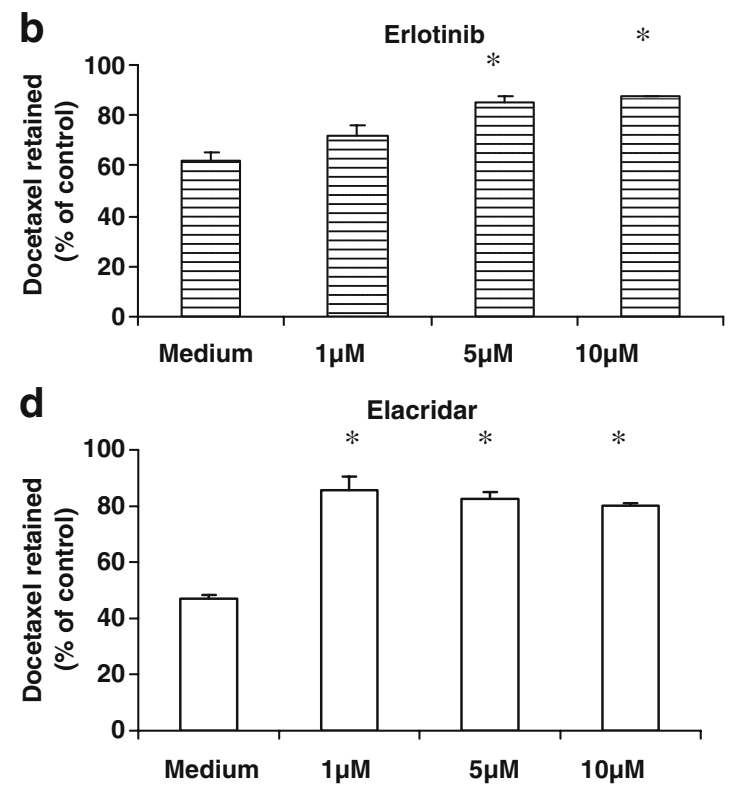

tyrosine kinase inhibitor (TKI) or elacridar in medium for $40 \mathrm{~min}$. Each result represents the average of three determinations. All inhibitors dissolved in DMSO. (* significant, $P<0.05$ relative to control) 
A) $2 \mu \mathrm{M} \mathrm{EPI}$

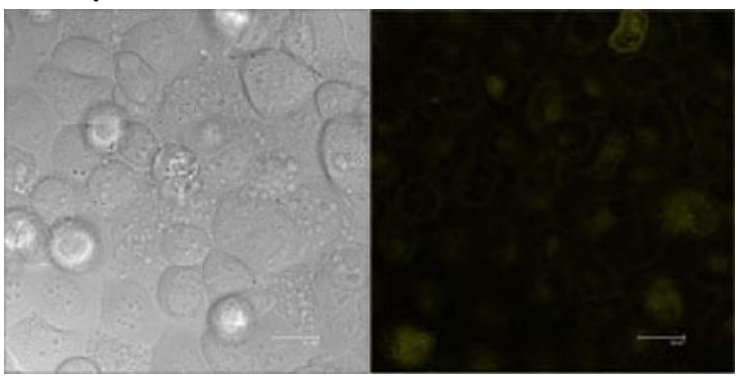

\section{C) $2 \mu \mathrm{M} \mathrm{EPI}+5 \mu \mathrm{M}$ Erlotinib}

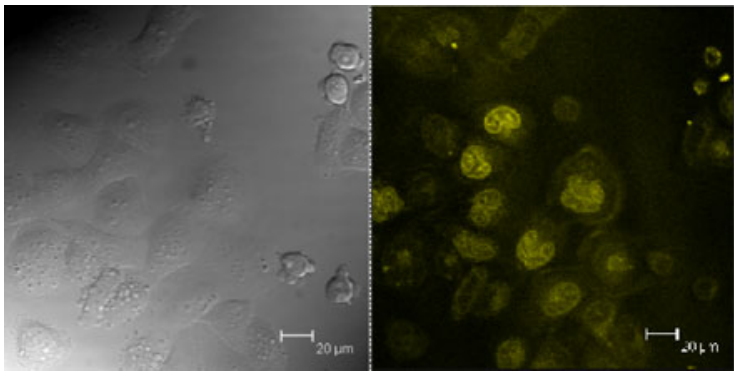

\section{E) $2 \mu \mathrm{M} \mathrm{EPI}+5 \mu \mathrm{M}$ Elacridar}

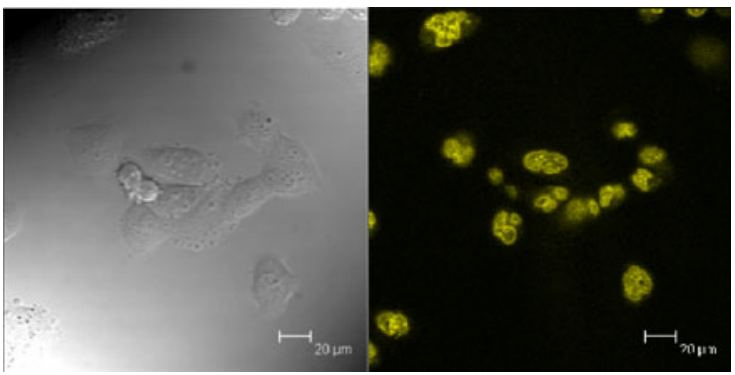

Fig. 5 Laser confocal imaging of epirubicin (EPI) accumulation in DLKP-A. A) EPI accumulation in DLKP-A in the absence of TKI over $120 \mathrm{~min}$. Epirubicin accumulation was visualised in the presence of $5 \mu \mathrm{M}$ lapatinib (b), erlotinib (c), gefitinib (d) and elacridar. (e). EPI

\section{B) $2 \mu \mathrm{M} \mathrm{EPI}+5 \mu \mathrm{M}$ Lapatinib}

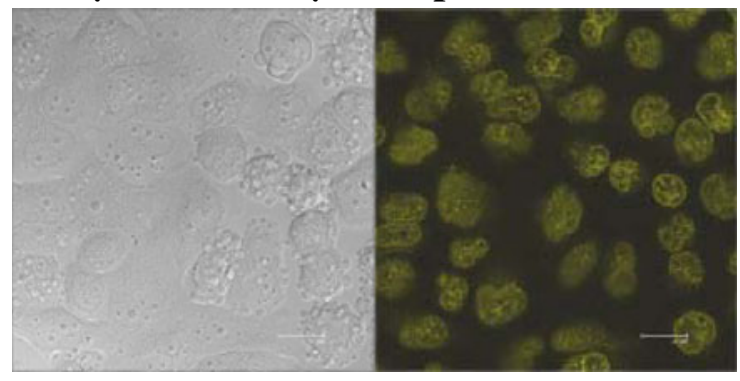

D) $2 \mu \mathrm{M} \mathrm{EPI}+5 \mu \mathrm{M}$ Gefitinib

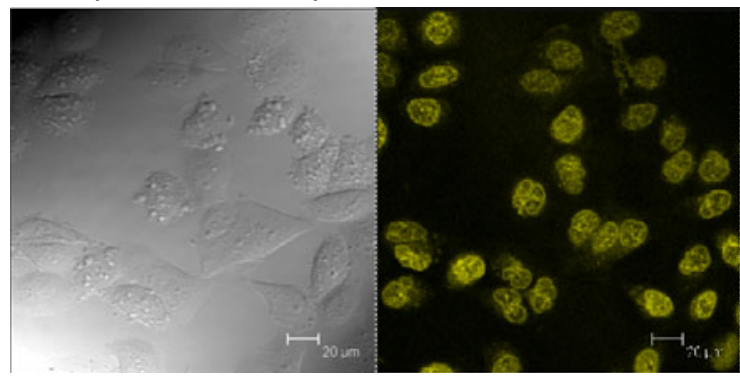

F) $2 \mu \mathrm{M}$ EPI + DMSO

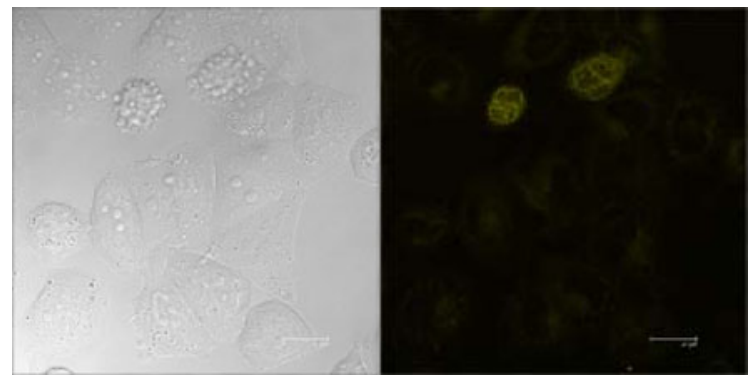

accumulation in the presence of $1 \%$ DMSO was included as a control (f). Each panel consists of a polarised light image on the left and the fluorescence image on the right. Scale bar represents $20 \mu \mathrm{m}$

Table 2 Combination proliferation results in DLKP and DLKP-A determined from 7-day assays

\begin{tabular}{|c|c|c|c|c|c|c|c|c|c|c|c|c|}
\hline & & \multicolumn{3}{|c|}{ Lapatinib $(\mu \mathrm{M})$} & & \multicolumn{3}{|c|}{ Gefitinib $(\mu \mathrm{M})$} & & \multicolumn{3}{|c|}{ Erlotinib $(\mu \mathrm{M})$} \\
\hline & & 0.125 & 0.25 & 1 & & 0.25 & 1 & 5 & & 0.125 & 0.5 & 2.5 \\
\hline DLKP & & $87 \pm 2$ & $85 \pm 3$ & $81 \pm 3$ & & $101 \pm 3$ & $97 \pm 1$ & $79 \pm 3$ & & $98 \pm 4$ & $95 \pm 4$ & $87 \pm 5$ \\
\hline $0.25 \mathrm{nM}$ Docetaxel & $74 \pm 4$ & $57 \pm 4$ & $58 \pm 3$ & $55 \pm 3$ & $57 \pm 3$ & $55 \pm 3^{\dagger}$ & $52 \pm 4^{\dagger}$ & $38 \pm 2$ & $76 \pm 8$ & $69 \pm 6^{\dagger}$ & $67 \pm 4^{\dagger}$ & $57 \pm 3$ \\
\hline 15nM Epirubicin & $50 \pm 2$ & $43 \pm 1$ & $41 \pm 2$ & $21 \pm 2$ & $73 \pm 1$ & $71 \pm 3^{\dagger}$ & $60 \pm 7^{\dagger}$ & $32 \pm 1$ & $73 \pm 4$ & $60 \pm 5$ & $38 \pm 3$ & $7 \pm 1$ \\
\hline DLKP-A & & $103 \pm 5$ & $100 \pm 5$ & $99 \pm 8$ & & $97 \pm 2$ & $91 \pm 9$ & $80 \pm 19$ & & $97 \pm 1$ & $93 \pm 6$ & $77 \pm 15$ \\
\hline 50nM Docetaxel & $88 \pm 4$ & $23 \pm 1$ & $3 \pm 0.2$ & $0.4 \pm 0.1$ & $63 \pm 2$ & $34 \pm 2$ & $3 \pm 0.2$ & $0.4 \pm 0.1$ & $61 \pm 1$ & $55 \pm 5^{\dagger}$ & $25 \pm 1$ & $1 \pm 0.1$ \\
\hline 750nM Epirubicin & $82 \pm 2$ & $51 \pm 5$ & $6 \pm 1$ & $2 \pm 0.2$ & $91 \pm 2$ & $76 \pm 8$ & $34 \pm 3$ & $5 \pm 0.3$ & $87 \pm 6$ & $84 \pm 1^{\dagger}$ & $73 \pm 1$ & $44 \pm 1$ \\
\hline
\end{tabular}

Values represent the average of three independent determinations. All values were significant except

${ }^{\dagger}$ values, not significant relative to cytotoxic proliferation value, $P>0.05$ and

${ }^{\dagger}$ values, not significant relative to TKI proliferation value, $P>0.05$ 
Table 3 Combination proliferation results in A549 and A549-Taxol determined from 7-day assays

\begin{tabular}{|c|c|c|c|c|c|c|c|c|c|c|c|c|}
\hline & & \multicolumn{3}{|c|}{ Lapatinib $(\mu \mathrm{M})$} & & \multicolumn{3}{|c|}{ Gefitinib $(\mu \mathrm{M})$} & & \multicolumn{3}{|c|}{ Erlotinib $(\mu \mathrm{M})$} \\
\hline & & 0.125 & 0.25 & 1 & & 0.25 & 1 & 5 & & 0.125 & 0.5 & 2.5 \\
\hline A549 & & $91 \pm 2$ & $87 \pm 2$ & $86 \pm 2$ & & $92 \pm 5$ & $88 \pm 7$ & $85 \pm 8$ & & $93 \pm 4$ & $84 \pm 6$ & $76 \pm 7$ \\
\hline $0.2 \mathrm{nM}$ Docetaxel & $97 \pm 1$ & $87 \pm 3^{\dagger \dagger}$ & $84 \pm 4^{\dagger \dagger}$ & $81 \pm 4^{\dagger \dagger}$ & $95 \pm 1$ & $80 \pm 4^{\dagger \dagger}$ & $74 \pm 4^{\dagger \dagger}$ & $64 \pm 3$ & $93 \pm 3$ & $82 \pm 7^{\dagger \dagger}$ & $73 \pm 5^{\dagger \dagger}$ & $61 \pm 5$ \\
\hline 20nM Epirubicin & $80 \pm 7$ & $55 \pm 5$ & $51 \pm 5$ & $30 \pm 4$ & $94 \pm 2$ & $68 \pm 5$ & $61 \pm 5$ & $39 \pm 5$ & $98 \pm 1$ & $87 \pm 3^{\dagger \dagger}$ & $69 \pm 2$ & $39 \pm 1$ \\
\hline A549-Taxol & & $92 \pm 2$ & $89 \pm 3$ & $88 \pm 2$ & & $89 \pm 2$ & $86 \pm 3$ & $81 \pm 3$ & & $87 \pm 5$ & $80 \pm 5$ & $78 \pm 5$ \\
\hline $0.75 \mathrm{nM}$ Docetaxel & $79 \pm 7$ & $32 \pm 4$ & $23 \pm 3$ & $17 \pm 2$ & $86 \pm 5$ & $52 \pm 9$ & $32 \pm 5$ & $22 \pm 2$ & $57 \pm 1$ & $44 \pm 2$ & $35 \pm 1$ & $17 \pm 1$ \\
\hline 30nM Epirubicin & $86 \pm 2$ & $65 \pm 4$ & $61 \pm 3$ & $46 \pm 1$ & $81 \pm 5$ & $61 \pm 4$ & $54 \pm 6$ & $35 \pm 3$ & $89 \pm 2$ & $64 \pm 2$ & $55 \pm 1$ & $33 \pm 1$ \\
\hline
\end{tabular}

Values represent the average of three independent determinations. All values were significant except

${ }^{\dagger}$ values, not significant relative to cytotoxic proliferation value, $P>0.05$ and

${ }^{\dagger}$ values, not significant relative to TKI proliferation value, $P>0.05$

Due to the absence of P-gp, TKI-docetaxel combinations did not produce supra-additive toxicity in DLKP (Table 2), or in A549 (Table 3). However, despite the absence of P-gp overexpression, epirubicin toxicity was potentiated in these cells, most notably by lapatinib and erlotinib co-incubation.

Effects of erlotinib and lapatinib on docetaxel accumulation in A549-Taxol

In order to examine the implications of the two different forms of TKI-P-gp interaction on docetaxel transport in a cell line expressing lower P-gp levels, A549-Taxol cells were exposed to $0.5 \mu \mathrm{M}{ }^{14} \mathrm{C}$ docetaxel in the presence of $0.25,1$, and $2.5 \mu \mathrm{M}$ erlotinib or lapatinib (Fig. 6) for $180 \mathrm{~min}$. A $1 \%$ DMSO control corresponding to the amount of DMSO present in the highest TKI concentration did not significantly affect the levels of ${ }^{14} \mathrm{C}$ docetaxel accumulated. Erlotinib, which the ATPase assays suggested was a P-gp substrate, and lapatinib, which was demonstrated to directly inhibit P-gp, both increased ${ }^{14} \mathrm{C}$ docetaxel accumulation in a time- and concentration-dependent manner. Lapatinib was more effective than erlotinib in altering drug accumulation in A549-Taxol. Lapatinib produced a significant increase in ${ }^{14} \mathrm{C}$ docetaxel at $0.25 \mu \mathrm{M}$, the lowest concentration examined and produced a greater effect at all concentrations examined.

\section{Discussion}

This study directly compared the interaction of three TKIs lapatinib, erlotinib and gefitinib with, primarily, the P-gp drug transporter while examining the effects the TKIs had on BCRP and MRP-1 ATPase activity. Our data indicates that there are important differences in the mechanism and potency of the interaction between the TKIs and the drug transporters.
The TKI ATPase assay results are generally consistent with previous findings but the comparative nature of the experiments revealed further information on the potential of each TKI to circumvent MDR in the absence of EGFR and HER-2. Gefitinib and erlotinib increased P-gp ATPase activation (Fig. 2a) but did not inhibit activated P-gp ATPase activity (Fig. 2b) indicating erlotinib to be a strong P-gp substrate while gefitinib, while also a substrate, is transported at a slower rate. The P-gp ATPase activation results are consistent with previous gefitinib and erlotinib data for this type of experiment while the P-gp ATPase inhibition observations have not previously been reported

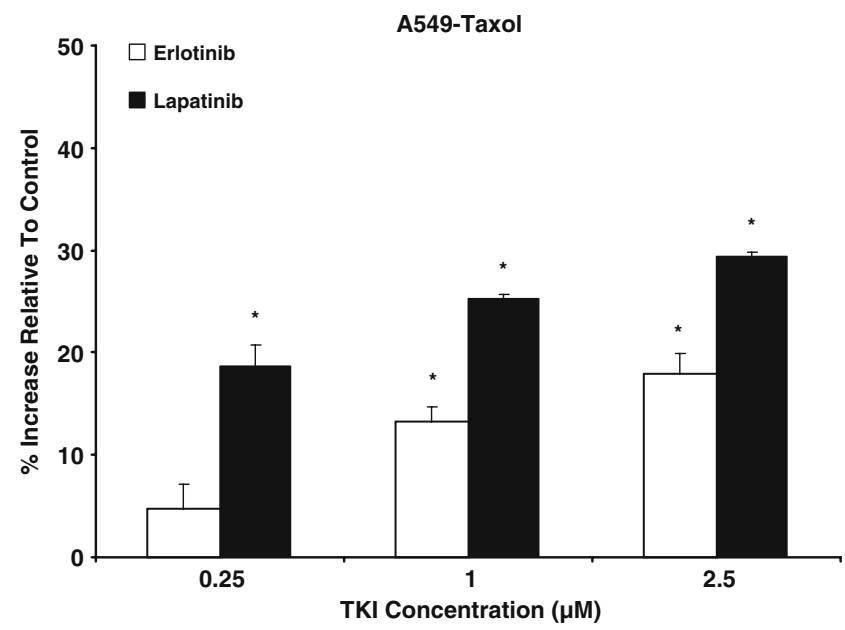

Fig. 6 The effects of erlotinib and lapatinib on accumulation of $0.5 \mu \mathrm{M}{ }^{14} \mathrm{C}$ radio-labelled docetaxel in the A549-Taxol cell line over $180 \mathrm{~min}$. Results are given as a percentage increase relative to the amount of ${ }^{14} \mathrm{C}$ radio-labelled docetaxel accumulated in A549-Taxol after $180 \mathrm{~min}$ in the absence of a P-gp modulatory agent (Control). Inclusion of a $1 \%$ DMSO control representing the highest amount of DMSO present in the assay showed no significant effect (results not shown). Each value represents the average of three determinations. All inhibitors dissolved in DMSO. (* significant, $P<0.05$ relative to control) 
$[14,17]$. Lapatinib is a poor activator of P-gp ATPase activity which suggests, when combined with the ATPase inhibition assay results, that it is a slowly transported substrate with a high affinity for a P-gp binding site. Lapatinib may therefore be more effective at overcoming P-gp-mediated resistance to chemotherapeutic agents than erlotinib and gefitinib, a point supported by the combination proliferation assay results (Table 2 and 3 ) in which lower concentrations of lapatinib were required to potentiate toxicity of P-gp substrate cytotoxics.

Along with P-gp, MRP-1 and BCRP are considered the key $\mathrm{ABC}$ proteins involved in multi-drug resistance. Gefitinib has a minor stimulatory effect on MRP-1 ATPase activity and no inhibitory effect on NEM-GS stimulated ATPase activity (Fig. 2c and supplementary Fig. 2), contrary to previous findings by Ozvegy-Laczka et al. [14]. MRP-1 requires the presence of glutathione to transport uncharged or mildly cationic compounds such as vincristine and etoposide [27]. It is therefore likely that the conflicting results may be attributable to the presence of glutathione $(2 \mathrm{mM})$ in the MRP-1 ATPase activation assay carried out in this project. There are no comparable MRP-1 ATPase activity studies for lapatinib and erlotinib published to date. All three TKIs exhibit minor ATPase activation suggesting possible interaction with MRP-1 as weak substrates in the presence of glutathione (Fig. 2c). Vincristine is a transported MRP-1 substrate but, consistent with the MRP-1 ATPase activation results in Fig. 2c, it does not increase MRP-1 ATPase activity [28]. The three TKIs did not inhibit NEM-GS stimulated MRP-1 ATPase activity up to a concentration of $30 \mu \mathrm{M}$ (Supplementary Fig. 2). Gefitinib has been shown to inhibit NEM-GS stimulated MRP-1 ATPase activity at higher concentrations that are not pharmacologically relevant [14]. Given these results, the presence of MRP-1 was unlikely to contribute to any of our TKI findings in the A549 and A549-Taxol cell lines used in this study (Fig. 1).

$\mathrm{BCRP}$ is a major drug resistance transporter that is modulated by the TKIs examined in this study [14]. The baseline vanadate-sensitive ATPase level in the mammalian cell BCRP membrane preparations used in this study are high enough to detect both activators and inhibitors of BCRP ATPase activity without need of a stimulating agent like verapamil in the P-gp ATPase inhibition assays [29]. The stimulation of BCRP ATPase activity by gefitinib is consistent with the results of Ozvegy-Laczka et al. although direct comparison is not possible due to the use of $S f 9$ insect membranes in their work, rather than the membranes of mammalian cell origin we employed (Fig. 2d) [14]. Low gefitinib concentrations stimulated BCRP ATPase activity while higher concentrations started to reduce the maximum BCRP ATPase activity. The concentration at which the TKIs inhibit the baseline ATPase activity is the point they become true inhibitors of BCRP ATPase. Lapatinib $(5 \mu \mathrm{M})$ inhibits the baseline BCRP ATPase activity at lower concentrations than gefitinib and does not achieve similar levels of ATPase stimulation (Fig. 2d). The analysis of BCRP ATPase activity suggests gefitinib and erlotinib have a greater affinity for BCRP at low concentrations than lapatinib (Fig. 2d). The decreased BCRP ATPase activity attributable to mitoxantrone was unexpected for a prototypical BCRP substrate. There is no comparable study of mitoxantrone in mammalian cell membranes to ascertain if this is a phenomenon associated with membrane preparations from differing origins or a characteristic of this particular substrate.

The results also indicate that the TKIs interact with BCRP at lower concentrations than P-gp, when considering stimulation of ATPase. Lapatinib is the poorest activator of both BCRP and P-gp ATPase activity. Further work is required to confirm if the TKIs are transported substrates at low concentrations.

ATPase assays alone do not definitively classify compounds as P-gp substrates or inhibitors. In vitro docetaxel transport assays confirmed lapatinib as the most potent Pgp modulator of the TKIs tested. Using a therapeutically relevant concentration of radiolabelled docetaxel, we showed each of the TKIs increased the net accumulation of docetaxel in the P-gp-over-expressing DLKP-A cell line (Fig. 3). The near maximal P-gp inhibition of docetaxel transport produced by lapatinib occurred at concentrations within reported peak serum levels [26].

Although cyclosporin A was a better inhibitor of verapamil-stimulated P-gp activity in the purified ATPase assay (Fig. 2), lapatinib was more effective at increasing Pgp-mediated docetaxel accumulation in DLKP-A (Fig. 3). The existence of more than one binding site for substrates and inhibitors, as reported for BCRP and P-gp, can mean complex interaction profiles when a modulatory agent is utilised $[3,30]$. This result highlights that the modulation of P-gp transport by a compound may vary depending on the P-gp substrate being investigated.

$\mathrm{IC}_{50}$ determinations in the NSCLC cell lines revealed little influence of P-gp on TKI efficacy, consistent with previous findings by Kitazaki et al. [16]. The high concentrations of TKIs that were needed to produce toxicity in these cells is likely to be due to the low (target) growth factor receptor expression levels in these cells and the observed toxicity is likely the result of a more non-specific toxic effect. Lapatinib proved to be the most potent TKI in all NSCLC cell lines, irrespective of EGFR and HER-2 status. Proliferation assays combining cytotoxic P-gp substrates and TKIs in the cells with low (A549-Taxol) and high P-gp overexpression (DLKP-A) both yielded strong cytotoxic synergy. The potentiation of epirubicin toxicity by the TKIs in A549-Taxol and DLKP-A was to be expected and 
the reason for it was graphically illustrated in the fluorescent laser confocal images in Fig. 5 showing increased nuclear localisation of epirubicin due to TKI treatment. TKI potentiation of epirubicin toxicity in the parental, non-P-gp expressing, A549 and DLKP cell lines was unanticipated. Inhibition of other putative epirubicin efflux mechanisms and interactions between pathways associated with tyrosine kinase-mediated signalling pathways and epirubicin toxicity may explain these observations. The effect is unlikely to be EGFR or MRP-1-related due to this potentiation being evident in the EGFR-negative DLKP cell line and the minor effect the TKIs have on MRP-1 ATPase activity.

The very high levels of P-gp present in DLKP-A make this an ideal cellular model in which to study the effects of putative P-gp inhibitors on P-gp substrate transport. While it is difficult to quantitatively compare the level of P-gp overexpression in these cells with that of typical clinical specimens, the lower over-expression of P-gp in A549Taxol cells may provide a model with levels of docetaxel resistance closer to that observed in vivo [31]. Erlotinib and lapatinib were selected for specific analysis as they represented the most effective P-gp ATPase activator and inhibitor, respectively, of the three TKIs examined. Both agents increased the accumulation of a physiologically relevant concentration of docetaxel $(0.5 \mu \mathrm{M})$ in the A549Taxol cell line, over a time period within the typical clinical serum drug exposure profile of both docetaxel [32] and each TKI. Lapatinib proved more potent than erlotinib at the clinically relevant concentrations examined.

Prolonged lapatinib-mediated P-gp inhibition might therefore be expected to affect the pharmacodynamic profiles of concurrently administered P-gp substrate cytotoxics (such as docetaxel or paclitaxel) by reducing P-gp mediated drug clearance from the body. This effect would be most pronounced in compartments in the body which are especially dependent on P-gp to provide protection from the toxic effects of drugs, e.g. certain stem cell populations such as those of the gut and blood [33, 34]. While a phase III study combining paclitaxel and lapatinib versus paclitaxel improved clinical outcomes in HER-2 positive breast cancers, a number of specific adverse interactions were reported for the lapatinib arm of the trial [35].

In conclusion, our data indicate that gefitinib and erlotinib, interact with P-gp as substrates and can inhibit P-gp-mediated transport of cytotoxic agents competitively. The distinct manner of the interaction of lapatinib with Pgp, suggest it is a poor substrate, and therefore a superior pump inhibitor, when compared to gefitinib and erlotinib. Combined with the transport and proliferation data, our findings suggest that P-gp over-expression is unlikely to generate resistance to lapatinib but may lead to gefitinib and erlotinib resistance. The TKIs have minimal interaction with MRP-1 but have a major effect on BCRP ATPase activity. Our results suggest an application for the TKIs, particularly lapatinib, as MDR modulators in P-gp-positive tumours in combination with P-gp substrate chemotherapy agents irrespective of EGFR and HER-2 status. Anthracycline/TKI combinations may be capable of additional anticancer actions, due to potentiation of toxicity even in the cell lines with little or no measurable P-gp activity. Lapatinib mono- and combination therapies may be useful in the treatment of NSCLC, where taxane combinations are emerging as standard first line therapies for advanced NSCLC but the pharmacokinetic inplications of combining TKIs with Pgp and BCRP substrate cytotoxics should be considered.

We propose that the combination of lapatinib with a taxane or an anthracycline warrants further clinical investigation.

Acknowledgements Lapatinib and Elacridar were kindly provided by GlaxoSmithKline. This work was made possible by a PRTLI cycle III award from the Higher Education Authority and funding from Cancer Research Ireland (CRI 03OCO).

Conflict of interest statement The authors have no conflicts of interest arising from the content of this manuscript.

\section{References}

1. Ito $\mathrm{K}$ et al (2005) Apical/basolateral surface expression of drug transporters and its role in vectorial drug transport. Pharm Res 22 (10):1559-1577. doi:10.1007/s11095-005-6810-2

2. Fardel O, Lecureur V, Guillouzo A (1996) The P-glycoprotein multidrug transporter. Gen Pharmacol 27(8):1283-1291. doi:10.1016/S0306-3623(96)00081-X

3. Loo TW, Clarke DM (2005) Recent progress in understanding the mechanism of P-glycoprotein-mediated drug efflux. J Membr Biol 206(3):173-185. doi:10.1007/s00232-005-0792-1

4. van der Deen $M$ et al (2005) ATP-binding cassette (ABC) transporters in normal and pathological lung. Respir Res 6:59. doi:10.1186/1465-9921-6-59

5. Modok S, Mellor HR, Callaghan R (2006) Modulation of multidrug resistance efflux pump activity to overcome chemoresistance in cancer. Curr Opin Pharmacol 6(4):50-54. doi:10.1016/j.coph.2006.01.009

6. Keri G, Orfi L (2006) Signal transduction therapy with rationally designed kinase inhibitors. Curr Signal Transduct Ther 1:67-95. doi: $10.2174 / 157436206775269190$

7. Roskoski R Jr (2004) The ErbB/HER receptor protein-tyrosine kinases and cancer. Biochem Biophys Res Commun 319(1):1-11. doi:10.1016/j.bbrc.2004.04.150

8. Kamath S, Buolamwini JK (2006) Targeting EGFR and HER-2 receptor tyrosine kinases for cancer drug discovery and development. Med Res Rev 26(5):569-594. doi:10.1002/med.20070

9. Dei Tos AP, Ellis I (2005) Assessing epidermal growth factor receptor expression in tumours: what is the value of current test methods? Eur J Cancer 41(10):1383-1392. doi:10.1016/j.ejca.2005.03.018

10. Dancey JE, Chen HX (2006) Strategies for optimizing combinations of molecularly targeted anticancer agents. Nat Rev Drug Discov 5(8):649-659. doi:10.1038/nrd2089

11. Kris MG (2005) How today's developments in the treatment of nonsmall cell lung cancer will change tomorrow's standards of care. Oncologist 10(Suppl 2):23-29. doi:10.1634/theoncologist. 10-90002-23 
12. Geyer CE et al (2006) Lapatinib plus capecitabine for HER2positive advanced breast cancer. N Engl J Med 355(26):27332743. doi: $10.1056 /$ NEJMoa064320

13. Yang $\mathrm{CH}$ et al (2005) Gefitinib reverses chemotherapy resistance in gefitinib-insensitive multidrug resistant cancer cells expressing ATP-binding cassette family protein. Cancer Res 65(15):69436949. doi:10.1158/0008-5472.CAN-05-0641

14. Ozvegy-Laczka C et al (2004) High-affinity interaction of tyrosine kinase inhibitors with the ABCG2 multidrug transporter. Mol Pharmacol 65(6):1485-1495. doi:10.1124/mol.65.6.1485

15. $\mathrm{Li} \mathrm{J}$ et al (2007) Association of variant ABCG2 and the pharmacokinetics of epidermal growth factor receptor tyrosine kinase inhibitors in cancer patients. Cancer Biol Ther 6(3):432438. doi:10.1158/1535-7163.MCT-06-0643

16. Kitazaki $\mathrm{T}$ et al (2005) Gefitinib, an EGFR tyrosine kinase inhibitor, directly inhibits the function of P-glycoprotein in multidrug resistant cancer cells. Lung Cancer 49(3):337-343. doi:10.1016/j.lungcan.2005.03.035

17. Shi $Z$ et al (2007) Erlotinib (Tarceva, OSI-774) antagonizes ATPbinding cassette subfamily B member 1 and ATP-binding cassette subfamily G member 2-mediated drug resistance. Cancer Res 67 (22):11012-11020. doi:10.1158/0008-5472.CAN-07-2686

18. Dai CL et al (2008) Lapatinib (Tykerb, GW572016) reverses multidrug resistance in cancer cells by inhibiting the activity of ATPbinding cassette subfamily B member 1 and G member 2. Cancer Res 68(19):7905-7914. doi:10.1158/0008-5472.CAN-08-0499

19. Duffy CP et al (1998) Enhancement of chemotherapeutic drug toxicity to human tumour cells in vitro by a subset of nonsteroidal anti-inflammatory drugs (NSAIDs). Eur J Cancer 34 (8):1250-1259. doi:10.1016/S0959-8049(98)00045-8

20. Clynes $M$ et al (1992) Multiple drug-resistance in variant of a human non-small cell lung carcinoma cell line, DLKP-A. Cytotechnology 10(1):75-89. doi:10.1007/BF00376102

21. Sarkadi B et al (1992) Expression of the human multidrug resistance cDNA in insect cells generates a high activity drugstimulated membrane ATPase. J Biol Chem 267(7):4854-4858

22. Martin A, Clynes M (1993) Comparison of 5 microplate colorimetric assays for in vitro cytotoxicity testing and cell proliferation assays. Cytotechnology 11(1):49-58. doi:10.1007/ BF00749057

23. O'Connor R et al (2007) A phase I clinical and pharmacokinetic study of the multi-drug resistance protein-1 (MRP-1) inhibitor sulindac, in combination with epirubicin in patients with advanced cancer. Cancer Chemother Pharmacol 59(1):79-87. doi:10.1007/ s00280-006-0240-7
24. Herbst RS et al (2002) Selective oral epidermal growth factor receptor tyrosine kinase inhibitor ZD1839 is generally welltolerated and has activity in non-small-cell lung cancer and other solid tumors: results of a phase I trial. J Clin Oncol 20(18):38153825. doi: $10.1200 / \mathrm{JCO} .2002 .03 .038$

25. Hidalgo $\mathrm{M}$ et al (2001) Phase I and pharmacologic study of OSI774 , an epidermal growth factor receptor tyrosine kinase inhibitor, in patients with advanced solid malignancies. J Clin Oncol 19 (13):3267-3279

26. Burris HA III et al (2005) Phase I safety, pharmacokinetics, and clinical activity study of lapatinib (GW572016), a reversible dual inhibitor of epidermal growth factor receptor tyrosine kinases, in heavily pretreated patients with metastatic carcinomas. J Clin Oncol 23(23):5305-5313. doi:10.1200/JCO.2005.16.584

27. Kruh GD et al (2001) MRP subfamily transporters and resistance to anticancer agents. J Bioenerg Biomembr 33(6):493-501. doi:10.1023/A:1012827221844

28. Hooijberg JH et al (2000) The effect of glutathione on the ATPase activity of MRP1 in its natural membranes. FEBS Lett 469(1):4751. doi:10.1016/S0014-5793(00)01238-2

29. Glavinas $\mathrm{H}$ et al (2007) ABCG2 (breast cancer resistance protein/mitoxantrone resistance-associated protein) ATPase assay: a useful tool to detect drug-transporter interactions. Drug Metab Dispos 35(9):1533-1542. doi:10.1124/dmd. 106.014605

30. Ozvegy-Laczka C et al (2005) Single amino acid (482) variants of the ABCG2 multidrug transporter: major differences in transport capacity and substrate recognition. Biochim Biophys Acta 1668 (1):53-63. doi:10.1016/j.bbamem.2004.11.005

31. Leonard GD, Fojo T, Bates SE (2003) The role of ABC transporters in clinical practice. Oncologist 8(5):411-424. doi:10.1634/theoncologist.8-5-411

32. Clarke SJ, Rivory LP (1999) Clinical pharmacokinetics of docetaxel. Clin Pharmacokinet 36(2):99-114. doi:10.2165/ 00003088-199936020-00002

33. Licht T et al (1994) P-glycoprotein-mediated multidrug resistance in normal and neoplastic hematopoietic cells. Ann Hematol 69 (4):159-171. doi:10.1007/BF02215949

34. Chen Z et al (1999) Multidrug resistance P-glycoprotein function of bone marrow hematopoietic cells and the reversal agent effect. J Tongji Med Univ 19(4):260-263

35. Di Leo A et al (2008) Phase III, double-blind, randomized study comparing lapatinib plus paclitaxel with placebo plus paclitaxel as first-line treatment for metastatic breast cancer. J Clin Oncol 26 (34):5544-5552. doi:10.1200/JCO.2008.16.2578 